مجلَّة جامعة مِصْرَ لللِّراساتِ الإنسانيَّة (اللُّغويَّات والآداب) مجلد 2 عدد 1 يناير (2022)

Analisi del discorso del romanzo di "Ouer pasticciaccio brutto de via Merulana" di C. E.

Gadda

'Uno studio stilistico-linguistico'

Analysis of the speech of the novel "Quer

pasticciaccio brutto de via Merulana" by C. $\mathrm{E}$.

Gadda

'A stylistic-linguistic study'

Nagwa Kassem*

nagwa.kassem@must.edu.eg

\title{
Abstract:
}

Carlo Emilio Gadda utilizza un linguaggio particolarissimo, che nasce dalla commistione di più elementi: lingue speciali, dialetti, linguaggio standardizzato, uso di termini tecnico-scientifici, ecc. Questo articolo prende in esame i tipi di discorso presenti nel romanzo di "Quer pasticciaccio brutto de via Merulana" di Carlo Emilio Gadda attraverso una classificazione presentata da una descrizione schematica e approssimativa.

*Italian Department, Faculty of languages \&amp; Translation , Misr University for Science \&amp; Technology, Egypt. 
I dialoghi e le espressioni riportate nel romanzo compaiono in discorso diretto,discorso indiretto libero, esposizione in romanesco,narrazione in italiano e narrazione mista.

\section{Parole chiave:}

\section{Gadda, Pasticciaccio, analisi del discorso, stile, linguaggio, plurilinguismo.}

\section{Abstract:}

Carlo Emilio Gadda uses a very particular language, which arises from the mixture of multiple elements: special languages, dialects, standardized language, use of technicalscientific terms, etc..This article examines the types of discourse present in the novel of "Quer pasticciaccio brutto de via Merulana" by Carlo Emilio Gadda through a classification presented by a schematic and approximate description. The dialogues and expressions reported in the novel appear in direct speech, free indirect speech, exposure in Roman dialect, narration in Italian and mixed narration.

The novel is very rich in terms belonging to sectoral languages, used in different ways and with different functions, offering a hint of the great variety of disciplines of which Gadda exploits the scientific terminology, which invades the whole novel. The research also investigates the ingredients of a wisely constructed and manipulated language 


\section{مجلَّة جامعة مِصْرَ للإِراساتِ الإنسانيَّة (اللُّويَّات والآداب) مجلد 2 عدد 1 يناير (2022)}

of Gaddian writing that serve to understand the technique of his experimentalism.

\section{Keywords:}

Gadda, Pasticciaccio, discourse analysis, style, language, multilingualism.

\section{Introduzione:}

Quer pasticciaccio brutto de via Merulana è un romanzo fondamentale nella ricca produzione di Carlo Emilio Gadda soprattutto per la sua lingua, che è un esempio eccezionale di plurilinguismo. Gadda mesola termini tecnici, elementi letterari, e forme gergali, creando così un linguaggio tutto suo.

Il motivo che mi ha stimolato ad interessarmi di Carlo Emilio Gadda scaturisce dalla lettura del suo particolare romanzo Quer Pasticciaccio brutto de via Merulana pubblicato nella sua stesura definitiva nel 1957 presso l'editore Garzanti.

Quer pasticciaccio brutto de Via Merulana è un romanzo avvenuto nei primi anni del fascismo nel 1927. È un giallo "aperto" che sta senza soluzione e venne pubblicato in volume nel 1957 dall'editore Garzanti. Considerato il 
capolavoro assieme a La cognizione del dolore (1963), e tradotto in tutto il mondo.

Leonardo Sciascia disse che Quer pasticciaccio brutto de via Merulana era «il più assoluto giallo che sia mai stato scritto: un 'giallo' senza soluzione» (Pieri 2011: 52). Presenta una particolare complessità stilistica in quanto sovrappone i vari livelli linguistici alti e bassi ai più vari lessici.

Si nota il titolo emblematico del romanzo 'Quer Pasticciaccio brutto de via Merulana' offre alcune indicazioni: la parlata è romanesca ("quer" per "quel", "de" per "di"); l'azione si svolge a Roma (Via Merulana); si tratta di un "Pasticciaccio" "brutto". Si osservi il suffisso peggiorativo -accio, seguito dall'elemento "brutto" che rafforza la connotazione, descrivendo l'avvenimento centrale con un ironico disprzzo come «pasticciaccio brutto».

Gadda seleziona di utilizzare il termine pastiche che $\mathrm{i}$ critici (primo fra tutti Gianfranco Contini) avevano utilizzato fin dai primi anni Trenta per descrivere il suo particolare stile. I dialetti sono interrotti da citazioni dal latino, dal greco antico, dal francese e dal tedesco e da un uso frequente di onomatopee. Pastiche riferisce non solo alla lingua utilizzata dallo scrittore, ma anche alla sua visione del mondo.

Contini (1989: 34) classifica lo stile di Gadda come pastiche, cioè come mescolanza di elementi eterogenei: voci 
dotte e letterarie, dialettismi, parole deformate, neologismi, linguaggi settoriali, gerghi, contaminazioni lessicali, «è la cifra gaddiana che più caratterizza il suo stile e la sua concezione di vita» ${ }^{1}$.Una commistione stilistica di codici, di registri, di linguaggi settoriali, di gerghi, di echi letterari, di contaminazioni lessicali, atti ad ottenere una rappresentazione straniata e umoristica della realtà.

Gadda (1993: 30) parla, in un passo menzionato in un'intervista, delle scelte linguistiche compiute nel Pasticciaccio riferendo di fatto alla categoria dell'italiano regionale, anche se naturalmente senza definirla rigorosamente:

Quanto al romanesco, non intendevo scodellare il vero e proprio dialetto; ma l'italiano misto a dialetto, quel modo vigoroso di parlare che hanno quelli che provengono per famiglia da un ambiente dialettale. [...] In sostanza, si tratta di una "contaminazione" tra italiano corrente e romanesco.

Gadda usa un linguaggio particolarissimo, che scaturisce dalla mescolanza di più molteplici elementi: linguaggi speciali, dialetti, linguaggio quotidiano standardizzato, utilizzo di termini tecnico-scientifici, gerghi, vocaboli arcaici

${ }^{1}$ Vedi Progetto di Letteratura italiana- "Voci del Novecento", anno scolastico 2007/08, disponibile a https://copernico.prato.it. 
e aulici, latinismi, grecismi, utilizzo di un linguaggio includente parole straniere (inglesi, francesi, spagnole, e tedesche), e neologismi inventati dallo scrittore stesso.

\section{La lingua di Gadda}

Non si può parlare di Gadda, del tessuto così stravagante e manipolato della sua scrittura, senza accennare agli ingredienti di un linguaggio sapientemente costruito.

Un'analisi, anche elementare, della composizione linguistica della prosa gaddiana può servire a capire la tecnica del suo sperimentalismo, e quanto egli abbia saputo far tesoro di ogni esperienza per arricchirlo e variarlo su più ampie reti stilistiche. In tal senso i dialettalismi, su cui troppo si è scritto, non sono che una delle componenti dell'impasto gaddiano, e neppure la più trasparente, strettamente connessa e interdipendente a tutte le altre. I principali nuclei dialettali in Gadda sono: lombardismi (in genere nelle opere milanesi); romaneschismi (soprattutto nel "Pasticciaccio"); fiorentinismi (specie nelle "Favole", in parte, in "Eros $e$ Priapo", e nel "Pasticciaccio"), spesso coincidenti con forme antiche o antiquate della lingua letteraria. I tecnicismi pure costituiscono l'aspetto più peculiare della prosa gaddiana, usati sia nel loro senso specifico che in un senso traslato a forte carica metaforica.

Un'altra componente di più fruttuoso impiego e dosaggio è l'intensa congerie delle forme personali: neoformazioni a 
base latina o greca: bucinando (11), cinobalanico (73); forme suffissali: scaricabarilistico (62); o prefissoidali: autoghigliottinarsi (187); forme analogiche: audicolo (166), giallazio (162); verbi parasintetici: dekirkegaardizzare (122) o desostantivali: priapare (110); forme parascientifiche: criptorutto (158); giustapposizioni brachilogiche: culseduto (62), domicilioaggredita (156); perifrasi aggettivali: er 'tu mi stufi', cioè il fidanzato (48); calembours linguistici: Facta factorum (37); deformazioni fonomorfologiche: irpotesi (208), setticimia (222) ecc.

A ciò si aggiunga, l'uso più eccessivo di ogni variante linguistica (allotropi, sinonimi ecc.) e l'uso abbondante di rilevanti onomatopee, di foresterismi, gergalismi, latinismi, arcaismi, forme latine, incastonature di greco e parole straniere.

Tutto ciò un sistema che mette il linguaggio in crisi, giocando stilisticamente sulla collisione di vari piani (ad esempio: forma colta/forma popolare ecc.), che rivela la volontà di Gadda di aggredire, oltre la realtà, la lingua stessa nei suoi segni, le possibilità della comunicazione.

Comunque la colorita commistione del linguaggio gaddiano, e il plurilinguismo dello scrittore, precisano le motivazioni e le funzionalità polemico-critiche che stanno alla base del suo sperimentalismo.

La sperimentazione linguistica di Gadda ha aiutato a riprendere l'Italia dialettale e ha stimolato agli esperimenti di 


\section{مجلَّة جامعة مِصْرَ للإِراساتِ الإنسانيَّة (اللُّويَّات والآداب) مجلد 2 عدد 1 يناير (2022)}

vari scrittori che si rifanno al fondo regionale, è importante ricordare oltre il "gergo" usato nel romanzo ha delle derivazioni culturali e un diverso significato morale.

Il lessico è certamente il fatto più facilmente osservato e descritto nella lingua del Pasticciaccio. Il romanzo accoglie parole straniere, parole dialettali e parole italiane; regionalismi e termini pan-italiani; l'italiano formale, il colloquiale e il triviale; l'italiano «settoriale» scientifico, quello tecnico, e quello burocratico.

Sono frequenti le parole e frasi in lingua straniera in tutte le opere di Gadda. Sono presenti alcuni termini in inglese: holding (39), minding (144), cost insurance free (186), flint (196), tight (38), vegetables (24), pipe-line (59), clacson (161), shampoo (163), boxer (105), pointer (149), cracking (122), free along bank (186), bulldog/bull-dog (92). È attinta la parola toboga (228) dall'inglese americano.

Sono presenti anche termini e locuzioni in francese: $a u$ ralenti (131), du vieux terroir (204), anisette (61), en passant (193), caveau (75), élite (69), bleu (15), dessous (41), loisir (128), trop-plain (221), refrain (131), coûte que coûte (73), foulard, foulards (15), bouquet (158), chez nous (73), bonbons (189), pensif (144), haute pâte (160), béchamelle (187), empâtée (204), nuits de Saint Petersbourg (87), cherchez la femme (4), taxì (63), l'espace d'un matin (64), mi-carême (158), hélas (183), loisir de siéger (133), manicure (88), négligé (15). 
Il tedesco si ritrova anche in discorsi diretti: ' $I l$ cannocchialante foca s'era creduto allora in dovere di riferire all'amministrazione - "Verwaltung, Verwal- tung!... Wo ist denn die Verwaltung? drüben links? Ach so!...' (80); "“Jedes Jahr ein Kind, jedes Jahr ein Kind..." gli cantava quel tedesco, ad Anzio: che pareva una foca» (10); o nella citazione di un proverbio: «keine Rose ohne Dornen» (183).

Alcuni vocaboli o espressioni in spagnolo completano il registro dei forestierismi del Pasticciaccio: cuidado (234), prensa (39), desde Italia (39), desde la misma Italia (39).

$\mathrm{Si}$ adoperano particolarmente, e con frequenza notevole, delle glosse metalinguistiche, come a p. 42: «Brutti caprari de la Sgurgola!».

Notiamo il verbo 'tegumentare': è un denominale ipertecnico (derivato dal biologico tegumento) trascurato dai dizionari attuali e non molto diffuso, per quanto di formazione chiara - marca una presa improvvisa di distanza del narratore dalla sua materia, quasi un segno di distacco scientifico.

Il Pasticciaccio contiene una materia linguistia molto variata e ricca che ha rinfrescato la lingua della narrazione del Novecento.Tale materia linguistica miscela molti ingredienti come: i linguaggi tecnici e scientifici, vari registri del parlato, il linguaggio quotidiano, i latinismi (ad hoc (213), agnus (150), ab aeterno (87), ad audiendum verbum (118), ad libitum (74), adnuente (adnuo) (103), asperges in 


\section{مجلَّة جامعة مِصْرَ للإِراساتِ الإنسانيَّة (اللُّويَّات والآداب) مجلد 2 عدد 1 يناير (2022)}

nomine Domini (71), coeli jucundum lumen et auras (187), consule (74), coram (202), Corpus Domini (113), crescite vero in gratia (167), do ut des (116), de jure decreto (161), de moribus, de temporibus (144), domum relapsa (166), Dominicus (34), evasi, effugi (84),), mescolati a parole di inventiva gaddiana creando quello che i francesi chiamano il pastiche.

A volte l'arricchimento del lessico appare attraverso la combinazione di termini ( ad esempio, composti come occipite-jungla (221), trubadorico-mandrillo (231), omaggio-sberleffo, mantiglia-vestaglia (15), duodeno-fegato (88), ecc.), e talvolta, in numero limitato di casi, attraverso l'invenzione di termini o la loro ricreazione semantica.

\section{Tipi di discorso:}

La voce del narratore cerca un dialogo continuo con il lettore: non è la voce decisiva di un narratore onnisciente, giudice infallibile del bene e del male, ma quella di chi esita, corregge giudizi, avanza ipotesi (di qui i vari «non si sa perché», «doveva di certo avercela», «s’intende», «sembravano banalità. Non erano banalità», «la causale principale, era sì, una. Ma..., direste oggi»). Questa colloquialità può sfociare nell'indiretto libero: la prima volta, riferendo i pensieri della vedova Antonini, che si risolvono in 


\section{مجلَّة جامعة مِصْرَ للإِراساتِ الإنسانيَّة (اللُّويَّات والآداب) مجلد 2 عدد 1 يناير (2022)}

un discorso diretto vero e proprio; la seconda volta, quando si riportano le obiezioni mosse a Ingravallo.

La voce narrativa, insomma, inizia a sfrangiarsi in voci diverse, composte, discordanti; perciò accoglie linguaggi eterogenei, che svariano dalle chiacchiere della vedova Antonini alle teorie di Aristotele, dal parlato quotidiano al tecnicismo legale. Quanto al dialetto, compare quasi esclusivamente nel discorso diretto; e non come dialetto puro. Antonini mescola italiano e romanesco; Ingravallo parla «contaminando napoletano, molisano e italiano». Le presenze dialettali nel testo narrativo sono appena tre: «gnommero», che ripete il pensiero di Ingravallo («Diceva anche...»); «e' femmene» che, ancora, è un'espressione del commissario; $\mathrm{i}$ «taliani» in ambito di indiretto libero: anche se, questa volta, non è possibile individuare un parlante preciso. Il dialetto, insomma, entra nel Pasticciaccio innanzitutto attraverso la strada naturalistica dell'imitazione di qualcuno; ma non può essere ridotto a questa pratica.

\subsection{Discorso Diretto:}

Frequentissimi sono i dialoghi e le espressioni riportati in stile diretto nel Pasticciaccio. Molti dei quali trasmessi interamente in romanesco. Quest'ultimo è assegnato non solo ai personaggi particolarmente di Roma, ma anche a quelli che provengono da altre zone del Lazio e i numerosi nativi dei Castelli Romani. 


\section{مجلَّة جامعة مِصْرَ للَّرِاساتِ الإنسانيَّة (اللُّويَّات والآداب) مجلد 2 عدد 1 يناير (2022)}

Quando il segno non è accompagnata da altre specificazioni (cioè in maggiore parte dei casi) significa che a parlare è uno di questi personaggi; al contrario, resa esplicita l'appartenenza di una battuta alla voce di personaggi italofoni (come il brigadiere Pestalozzi, piemontese, o il capufficio di Giuliano Valdarena, di cui non è rivelata la provenienza, non ricavabile dal suo modo di esprimersi), oppure a quella del commissario Ingravallo. È necessario notare che quest'ultimo, contrariamente a quanto si legge nel passo in cui viene presentato ai lettori, non sempre parla «contaminando napolitano, molisano, e italiano» (17), ma piuttosto spesso si serve anche di parole o frasi romanesche.

\section{Esempi del Discorso Diretto:}

- «Potevo annà in giro pe Roma co un preciutto in collo? [...] Pe sentì dì da la gente: avemo visto er commendator Angeloni a via Panisperna che arrancava co un caciocavallo in collo? co du fiaschi [...] che pareveno du gemelli, in collo a la balia?» (31).

- «Si m'ha dato du schiaffi, embè? ? è stato un affare tra de noi: nun lo ponno carcerà pe questo» (140).

- «Fàmese a crompà la porca, signori! «Fàmese a caccià li sordi, ch'è la vorta bona, signori! ch'è na vergogna lassalla qua sur banco che a momenti aripiove, che cioo so che ce $n$ 'avete un sacco in saccoccia, de baiocchi. Fama annà via la migragna, signori! La porca è vostra, si è che cacciate li baiocchi.»(218). 
Il narratore assume la caratteristica di un narratore naturalista, disegnando con estrema verosimiglianza la 'mimica e la gestualità' 2 di Ingravallo e riportando in discorso diretto le sue espressioni più vere, in quel misto di «napolitano, italiano e molisano: «Quanno me chiammeno!... Già. Si me chiammeno a me... può sta ssicure ch'è nu guaio: quacche gliuommero... de sberretà...» diceva, contaminando napolitano, molisano, e italiano.» (3).

\subsection{Discorso indiretto libero:}

Il Pasticciaccio presenta una produzione più notevole, e anche più riuscita, dello stile indiretto libero nella narrativa italiana.

Attraverso il discorso indiretto libero, sono molti i brani in cui il romanesco eccedere i dialoghi per entrare nella narrazione: "quattordici o quindici dei più autorevoli, de queli che t'abbruceno subito er gargarozzo, appena ingolli» (60).

Sicuramente attribuibili ad Ingravallo sono alcune espressioni qui presenti in stile indiretto libero, tipo il fattaccio, come si torce il collo a un pollo o sotto forma di

${ }^{2}$ Cfr. S. Bisanti, e M. Cecchini, Ubiquo ai casi: analisi della prima sequenza di Quer pasticciaccio brutto de via Merulana, pp. 3-4, disponibile a http://michelececchini.it/officina/ubiquo-ai-casi-analisidella-prima-sequenza-di-quer-pasticciaccio-brutto-de-via-merulana/ 


\section{مجلَّة جامعة مِصْرَ للإِراساتِ الإنسانيَّة (اللُّويَّات والآداب) مجلد 2 عدد 1 يناير (2022)}

discorso diretto o citazione: in questo caso si noterà che la contaminazione di italiano e dialetto costituisce il tratto caratteristico della lingua di don Ciccio ( $E$ poi pareva pentirsi, come d'aver calunniato 'e femmene) (4).

\section{Esempi del Discorso Indiretto Libero:}

- «Se sarebbe buttata sur tavolaccio: duro pe duro se sarebbe potuta addormì. Chissà che puro li parenti nun s'addormissino» (142).

- «una gran disinvoltura nello stirare $i$ pantaloni, dopo avenne affiarati un sei o sette para al Balducci» (110).

- «Del resto, ammappela! du fianchi in gloria du seni de marmo: du zinne toste» (111).

- «Ingravallo, similmente a certi nostri filosofi, attribuiva un'anima, anzi un'animaccia porca, a quel sistema di forze» (17).

\subsection{Esposizione in Romanesco:}

Si nota l'uso gaddiano, a lui specifico, del dialetto nelle sue opere. Il linguista Claudio Marazzini dice intorno all'utilizzo di Gadda del dialetto:

Un uso diverso del dialetto [...] negli scrittori 'mistilingui' come Carlo Emilio Gadda. Nella sua pagina si affollano i più vari elementi. Non c'è solo dialetto, ma una varietà: lombardo nell'Adalgisa e nella Cognizione del dolore, fiorentino nelle Favole 
e in Eros e Priapo, romanesco e molisano, con qualche battuta in veneto, in Quer pasticciaccio brutto de via Merulana. È il 'multilinguismo' o pastiche gaddiano: attraverso un processo di straniamento, materiali eterogenei convergono nella pagina dello scrittore, con esiti espressionistici. (Cfr. Marazzini 2004: 205-206).

Questo pastiche è una particolarità decisiva dello stile gaddiano; mescolanza di lingue, personaggi, e situazioni che risultano nel romanzo creando un'impressione barocca.

Con il dialetto romanesco si sente e appare la paura degli abitanti dopo il furto dei gioielli della signora Menegazzi. Tale paura si manifesta durante l'interrogazione di una ragazzina che forse aveva visto il responsabile del delitto:

Di', di' pupa mia bella! nun piagne, che co te ce sta mamma tua che te vo tanto bene, tiè,» le scoccò du baciozzi, «nun te spaventà der dottore. Er dottor Ingarballo nun è un dottore de queli brutti, che so' tanto cattivi, poveretti, de queli che te fanno la bua su la lingua. ¿̀ un dottore cor vestito nero, ma ̀̀ tanto bono!. (72).

In una scena del romanzo, descrivendo l'ambito del locale, il dialetto esce dall'ambito del discorso e infiltra di nuovo la narrazione stessa. Pare che all'improvviso Gadda inserisca delle frasi in dialetto come: 
«Er pollice l'aveva infilato ner buco d'una tavoletta e coll'artre dita l'istessa mano strigneva un mazzetto de pennelli, da spennellà co la tintura nun se sa che pezzo de pelle, si gnente gnente j'avesse trovato un quarche strugnoccolo, a quarchiduna». (124).

Una caratteristica particolare del Pasticciaccio è il fatto che il dialetto non è attribuito continuamente ad un personaggio. Matt (2012: 23) dice che in alcuni brani la narrazione procede utilizzando pienamente il romanesco, senza che ciò si possa spiegare con l'adozione del punto di vista di uno degli attori del dramma. In alcuni casi la voce narrante sembra lasciar posto a «un narratore sconosciuto, persona incolta, normalmente inserita nel mondo dialettale romanesco» (Cane 1969: 114). È ciò che capita, per citare solo il caso più notabile, nella descrizione del funerale di Liliana, in cui, «oltre che nell'uso del dialetto, la popolarità della voce narrante si manifesta nel modo semplicistico di vedere le cose» (Matt 2011: 192). In tutte le pagine di questo tipo, il discorso non è condotto integralmente in puro romanesco, ma è contaminato da continui frammenti di italiano. Il carattere di narrazione in romanesco indica un uso del dialetto non esclusivo, ma dominante, ad esempio: «du fojacci de bandone pe parafanghi ripitturati de nero cor pennello». (P. 223). 


\subsection{Narrazione in Italiano:}

Il segno di narrazione in italiano indica un discorso in cui l'italiano è nettamente prevalente, ciononostante il romanesco possa comunque comparire attraverso singole parole o espressioni: "le sue rare opinioni [...] le annotavano in un'agendina presto presto, da non lasciarne addietro un sol micolo». (39).

Ci sono, particolarmente nella seconda parte del romanzo, brani in cui il scenario è interamente esterno al mondo rappresentato, e la voce narrante pertanto non risulta influenzata con le voci dei personaggi. comunemente, in una prosa complessa come quella gaddiana è sempre possibile che un elemento estraneo faccia capolino nel discorso.

\section{Esempi della narrazione in Italiano:}

- «si sbronzava a Tivoli con un suo drudo macellaro: e ci voleva poi del bello e del buono perché i carabinieri non la rispedissero a Zagarolo con foglio di via obbligatorio: data "l'incapacità di sussistere coi propri mezzi» e data anche la fattispecie: pubblico scandalo. Il macellaro, non si capiva di preciso in che modo, riusciva a tacitarli ogni volta» (80).

- «Er Maccheronaro levò le palpebre serio serio, cor labbro tuttavia sporto un millimetro, affisando senza dir parola il cliente diletto, nel momento e nell'atto stesso che gli porgeva quel trofeo.» (134). 


\section{مجلَّة جامعة مِصْرَ للإِراساتِ الإنسانيَّة (اللُّويَّات والآداب) مجلد 2 عدد 1 يناير (2022)}

- «ambulavano a fatica da uno spaccio e da un ombrellaccio al successivo [...]: si rivolvevano, si strofinavano $i$ rispettivi gregori» $(220)$.

\subsection{Narrazione mista:}

Il segno di narrazione mista indica un discorso in cui italiano e romanesco sono compresenti, senza che uno dei due idiomi prevalga nettamente sull'altro. Si vede, per esempio: "facendo tuttodi le viste d'esser solo intento a razzolare, a cercar bachi. Certi bagarozzi, certi vermini» (200).

Molte zone del romanzo sono caratterizzate da una netta instabilità nel punto di vista adottato. Ne consegue una forte presenza di brani in cui si passa continuamente dall'italiano ipercolto tipico di Gadda al dialetto e viceversa.

L'alternanza di linguaggi può facilmente verificarsi in maniera molto brusca, anche all'interno di singole frasi, che cominciano in un codice e terminano in un altro (come si vede bene, per esempio, nella descrizione del mercato di Piazza Vittorio che fa da cornice all'arresto di Ascanio Lanciani). 


\section{Esempi della narrazione mista:}

- " "Un po' de stracchino? De quello de Corticelli che je piace tanto, dottó?" E, al grugno che mise: "Un pochetto solo, dottò! Cioo provi: è tanto bono!... Mica je po fa male...»" Sotto al riflettore di vetro, orlato di crespe e di riccioli bianchi e verdini come l'insalata, er cucuzzone pareva più tenebroso, più riccioluto del solito» (134).

- «Fino alla maggiore età della pupilla il malloppo doveva essere conferito, per l'amministrazione, a due curatori» (79).

- «mortadella cotta a fette alterne, mollemente adagiata in quel divano a opera dei diti peritissimi e paffutelli del Maccheronaro» (134).

La classificazione appena esposta nasce da una descrizione schematica e inevitabilmente approssimativa dei tipi di discorso presenti nel romanzo. La complessità delle scelte narrative compiute da Gadda, in particolare riguardo al problema del punto di vista, è tale da richiedere una trattazione ben più articolata: la plurivocità del Pasticciaccio è una macchina che andrebbe ricostruita in maniera estremamente analitica. Non sempre, per citare solo uno dei tanti punti controversi, la distinzione tra Discorso indiretto libero e Narrazione in romanesco è di semplice individuazione (Cfr. Matt 2012: 20).

Tuttavia, credo che la prova di classificazione qui 
compiuta possa avere una sua utilità pratica. In particolare, per tutte quelle parole che possono essere interpretate sia come romanesche sia come italiane, le marche di discorso attribuite a tutti i contesti paiono in grado di fornire indicazioni valide, anche se non sempre definitive, per una corretta interpretazione. 


\section{Conclusioni:}

Con questo studio cerco di analizzare, il più possibile nella loro interezza, i tipi di discorso presenti nel Pasticciaccio, studiando lo stile di Gadda: assumendo i tratti della sua varietà lessicale.

Attraverso queste pagine, possiamo allora concludere che Gadda, non ha fatto altro che avallare quanto espresso dal suo personaggio, di tanto in tanto fornendo alle riflessioni di quest'ultimo addirittura un più preciso impianto teorico e puntuali riferimenti. Egli, abbiamo visto, rappresenta il mondo proprio come un garbuglio, senza affatto attenuarne l'inestricabile complessità, la presenza simultanea degli elementi più eterogenei che concorrono a determinare ogni evento.

La mescolanza della lingua tradizionale letteraria con i dialetti, mescolati con i termini tecnico-specifici, era un metodo di Gadda per combattere la società alta e la sua lingua privata per creare una forma d'arte per il popolo.

Gadda rappresnta le vicende degli episodi della vita reale come un "groviglio inesplicabile" in balìa del caos. Egli vede il mondo come un garbuglio, un caos, un groviglio; o gnommero che alla romana vuol dire gomitolo; e per raffigurare questo pasticcio si usa di una speciale scrittura basata sulla commistione dei linguaggi. 


\section{مجلَّة جامعة مِصْرَ للَّرِاساتِ الإنسانيَّة (اللُّويَّات والآداب) مجلد 2 عدد 1 يناير (2022)}

\section{Bibliografia:}

- $\quad$ Cane, Eleonora. 1969. Il discorso indiretto libero nella narrativa italiana del Novecento, Silva, Roma.

- Contini, Gianfranco. 1989. Quarant'anni d'amicizia. Scritti su Carlo Emilio Gadda (1934-1988), Einaudi, Torino.

- Gadda, Carlo Emilio. 1957. Quer pasticciaccio brutto de via Merulana, Garzanti, Milano.

- Gadda, Carlo Emilio. 1993. L'apotema del mattone, in ID., «Per favore mi lasci nell'ombra» Intervista 1950-1972, a cura di Claudio Vela, Adelphi, Milano (pp.78-79).

- Isella, Dante. 2001. I cari 'latini' di Gadda, in 'I Quaderni dell'Ingengere. Testi e studi gaddiani' (già in «Aufidus»35, 1998, pp. 119-131).

- Matt, Luigi. 2006. Gadda. Storia linguistica italiana, Carocci, Roma.

- Matt, Luigi. 2010. Osservazioni sulla lessicografia romanesca, in «Studi di Lessicografia Italiana», Vol. 27 (pp. 153-184).

- $\quad$ Matt, Luigi. 2011. La narrativa del Novecento, il Mulino, Bologna.

- Matt, Luigi. 2012. Quer Pasticciaccio brutto de via Merulana, Glossario romanesco, Aracne, Roma. 


\section{مجلَّة جامعة مِصْرَ للَّرِاساتِ الإنسانيَّة (اللُّويَّات والآداب) مجلد 2 عدد 1 يناير (2022)}

- Paccagnella, Ivano. 1994. Uso letterario dei dialetti, in Storia della lingua italiana Serianni, a cura di L. Serianni \& P. Trifone, Einuadi, Torino, 3 voll., vol. $3^{\circ}$ "Le altre lingue" (pp. 465-539).

- $\quad$ Pieri, Giuliana. 2011. Italian Crime Fiction. European Crime Fictions, University of Wales Press, Cardiff.

- Porro, Marzio. 1973. I linguaggi della scienza e della tecnica, in Gian Luigi Beccaria (a cura di): I linguaggi settoriali in Italia, Bompiani, Milano, (pp. 181-206).

- Rovere, Giovanni. 1989. Sottocodici e registri in testi tecnici. Occorrenze e cooccorrenze, in Rivista italiana di Dialettologia Vol. 13 (pp.135-160).

- Rovere, Giovanni. 2010. Linguaggi settoriali, in Enciclopedia dell'Italiano (diretta da R. Simone). Roma. vol. I (pp. 804-806).

- Serianni, Luca \& Trifone, Pietro, (1994). (a cura di), Storia della lingua italiana. Vol. 3, Le altre lingue, Einaudi, Torino.

- Ungaretti, Giulio. 1993. Gadda al microfono. L'ingegnere e la Rai. 1950-1955, Nuova ERI, Torino. 


\section{Sitografia:}

- Progetto di Letteratura italiana- "Voci del Novecento", anno scolastico 2007/08, disponibile a https://copernico.prato.it.

- Bisanti, Sara \& Cecchini, Michele, Ubiquo ai casi: analisi della prima sequenza di Quer pasticciaccio brutto de via Merulana, disponibile a http://michelececchini.it/officina/ubiquo-ai-casi-analisidella-prima-sequenza-di-quer-pasticciaccio-brutto-devia-merulana/

- The Edinburgh Journal of Gadda Studies» (Cfr. :http://www.gadda.ed.ac.uk). 